\title{
THE FIRST DETECTION OF PHOTOMETRIC VARIABILITY IN A Y DWARF: WISE J140518.39+553421.3
}

\author{
Michael C. Cushing ${ }^{1}$, Kevin K. Hardegree-Ullman ${ }^{1}$, Jesica L. Trucks ${ }^{1}$, Caroline V. Morley ${ }^{2}$, John E. Gizis ${ }^{3}$, \\ Mark S. Marley ${ }^{4}$, Jonathan J. Fortney ${ }^{2}$, J. Davy Kirkpatrick ${ }^{5}$, Christopher R. Gelino ${ }^{4}$, \\ Gregory N. Mace ${ }^{6}$, And Sean J. Carey ${ }^{7}$ \\ ${ }^{1}$ The University of Toledo, 2801 West Bancroft Street, Mailstop 111, Toledo, OH 43606, USA; michael.cushing@utoledo.edu \\ ${ }^{2}$ Department of Astronomy and Astrophysics, University of California, Santa Cruz, CA 95064, USA \\ ${ }^{3}$ Department of Physics and Astronomy, University of Delaware, Newark, DE 19716, USA \\ ${ }^{4}$ NASA Ames Research Center, Moffett Field, CA 94035, USA \\ 5 Infrared Processing and Analysis Center, California Institute of Technology, Pasadena, CA 91125, USA \\ ${ }^{6}$ Department of Astronomy, The University of Texas, Austin, TX 78712, USA \\ ${ }^{7}$ Spitzer Science Center, California Institute of Technology, Pasadena, CA 91125, USA \\ Received 2015 July 24; accepted 2016 February 17; published 2016 June 1
}

\begin{abstract}
We present the first detection of the photometric variability in a spectroscopically confirmed Y dwarf. The Infrared Array Camera on board the Spitzer Space Telescope was used to obtain time series photometry of WISE $\mathrm{J} 140518.39+553421.3$ at 3.6 and $4.5 \mu \mathrm{m}$ over a $24-\mathrm{hr}$ period at two different epochs separated by 149 days. Variability is evident at $4.5 \mu \mathrm{m}$ in the first epoch and at 3.6 and $4.5 \mu \mathrm{m}$ in the second epoch, which suggests that the underlying cause or causes of this variability change on the timescales of months. The second-epoch [3.6] and [4.5] light curves are nearly sinusoidal in form, in phase, have periods of roughly $8.5 \mathrm{hr}$, and have semi-amplitudes of $3.5 \%$. We find that a simple geometric spot model with a single bright spot reproduces these observations well. We also compare our measured semi-amplitudes of the second-epoch light curves to predictions of the static, onedimensional, partly cloudy, and hot spot models of Morley and collaborators, and find that neither set of models can reproduce the observed [3.6] and [4.5] semi-amplitudes simultaneously. Therefore, more advanced twodimensional or three-dimensional models that include time-dependent phenomena like vertical mixing, cloud formation, and thermal relaxation are sorely needed in order to properly interpret our observations.
\end{abstract}

Key words: brown dwarfs - infrared: stars - stars: individual (WISE J140518.39+553421.3) - stars: low-mass

Supporting material: data behind figure

\section{INTRODUCTION}

Y dwarfs are the coolest class of brown dwarfs known (Cushing et al. 2011; Kirkpatrick et al. 2012), with estimated effective temperatures $\left(T_{\text {eff }}\right)$ below $500 \mathrm{~K}$ (e.g., Dupuy \& Kraus 2013). At such low temperatures, their photospheres are composed of $\mathrm{H}_{2}, \mathrm{He}, \mathrm{H}_{2} \mathrm{~S}, \mathrm{CH}_{4}, \mathrm{H}_{2} \mathrm{O}$, and $\mathrm{NH}_{3}$ in the gas phase, and salt $(\mathrm{KCl})$, sulfide $\left(\mathrm{MnS}, \mathrm{Na}_{2} \mathrm{~S}\right.$, and $\left.\mathrm{ZnS}\right)$, and possible water ice condensates in the solid phase, which gravitationally settle within the atmosphere to form clouds (Morley et al. 2012, 2014b). Y dwarfs are ideal analogs to the cool gas giant exoplanets predicted to be discovered by highcontrast imagers such as the Gemini Planet Imager (GPI; McBride et al. 2011) and the Spectro-Polarimetric Highcontrast Exoplanet REsearch (SPHERE; Beuzit et al. 2008) instrument on the Very Large Telescope. However, there are only 21 spectroscopically (and two photometrically) confirmed $\mathrm{Y}$ dwarfs known and since they are intrinsically very faint, with $M_{J} \gtrsim 20 \mathrm{mag}$, our understanding of their basic properties is still very limited.

The formation and subsequent evolution of condensate clouds plays a critical role in the evolution of all brown dwarfs. As a brown dwarf cools and passes through the MLTY sequence, various solid phase or liquid phase condensate clouds form until the atmosphere is eventually composed of layers upon layers of clouds, similar to the conditions seen in Jupiter. It has long been thought that inhomogenous cloud coverage might give rise to variations in the integrated intensity of a brown dwarf as it rotates (Ackerman \& Marley 2001; Burgasser et al. 2002). Photometric variability was originally detected in L dwarfs in the $I$ band by Bailer-Jones \& Mundt (2001) and Gelino et al. (2002), but has now been detected in both $\mathrm{L}$ and $\mathrm{T}$ dwarfs, at red-optical $(0.70-1.0 \mu \mathrm{m})$, nearinfrared $(1-2.5 \mu \mathrm{m})$, and mid-infrared $(2.5-5.0 \mu \mathrm{m})$ wavelengths, and both photometrically and spectroscopically (e.g., Enoch et al. 2003; Morales-Calderón et al. 2006; Buenzli et al. 2012; Khandrika et al. 2013; Radigan et al. 2014; Metchev et al. 2015). Interpretations of this variability range from simple holes in the clouds to variations in the thickness of uniform cloud decks. More recently, the potential for so-called "hot spots" or temperature variations giving rise to these variations has also been suggested (Showman \& Kaspi 2013; Morley et al. 2014a; Robinson \& Marley 2014).

Although variability is conspicuous in L and T dwarfs, no Y dwarf variability has been reported in the literature. We therefore initiated a Cycle 11 Spitzer Space Telescope Exploration Science program (90015, PI: Cushing) to search for photometric variability in $\mathrm{Y}$ dwarfs using the Infrared Array Camera (IRAC; Fazio et al. 2004) at 3.6 and $4.5 \mu \mathrm{m}$ (hereafter [3.6] and [4.5]). Fourteen Y dwarfs were observed continuously for roughly $24 \mathrm{hr} ; 12 \mathrm{hr}$ at [3.6], followed by $12 \mathrm{hr}$ at [4.5]. The observations were then repeated a few months later in order to search for any changes in the light curves. In this work, we present the detection of photometric variability in the Y0.5pec? ${ }^{8}$ dwarf WISE J140518.39+553421.3 (hereafter

\footnotetext{
8 WISE J1405+5534 was classified as Y0p? by Cushing et al. (2011) because the peak of the $H$-band emission was shifted redward by roughly 60 A relative to other late-type T dwarfs and early-type Y dwarfs. Schneider et al. (2015) later reclassified WISE J1405+5534 as Y0.5 but dropped the pec? by mistake.
} 
WISE J1405+5534; Cushing et al. 2011). A future paper will discuss the results from the entire survey.

\section{SPITZER OBSERVATIONS AND DATA REDUCTION}

WISE J1405+5534 was observed on 2013 March 22 (hereafter epoch 1) and 2013 August 17 (hereafter epoch 2) with IRAC at both [3.6] and [4.5]. The Spitzer Astronomical Observation Request numbers for these observations are 47166208, 47162624, 47173888, and 47169792. It was observed in the "staring mode" in which a target is held close to the same position on the array in order to minimize the effects that variations in the quantum efficiency across an individual pixel have on the resulting photometry (i.e., the pixel phase effect; Reach et al. 2005). WISE J1405+5534 was also positioned in the upper left corner of the detector at the "sweet spot" pixel so that we could use the high-resolution gain map of the sweet spot. During each epoch, a series of roughly 430 images, each with an exposure time of $100 \mathrm{~s}$, was obtained over a continuous 12-hr period in [3.6], followed by a 12-hr period in [4.5]. Images were also taken for 30 minutes prior to the start of the two [3.6] 12-hr sequences in order to mitigate initial telescope drift and allow for the spacecraft to settle. These data were ignored in our analysis.

We began our analysis using the Basic Calibrated Data (BCD) frames generated by the Spitzer Science Center using version S19.1.0 of the IRAC science pipeline. Before performing aperture photometry on the $\mathrm{BCD}$ images, we converted the images from units of $\mathrm{MJy} \mathrm{sr}^{-1}$ to total electrons by dividing the images by the flux conversion factor (the FLUXCONV FITS keyword) in units of MJy $\mathrm{sr}^{-1} / \mathrm{DN} \mathrm{s}^{-1}$, multiplying by the gain in units of electrons $\mathrm{DN}^{-1}$, and then multiplying by the exposure time in seconds. The remainder of the reduction was performed using custom Interactive Data Language (IDL) code. The position of WISE J1405+5534 was determined using the box_centroider routine with an aperture radius of 3 pixels and a background annulus between 3 and 7 pixels. Using the IDL aper routine with the EXACT keyword set we measured the total number of electrons detected within a 3 pixel radius of the centroid position. Background subtraction was accomplished by computing the mean number of electrons in an annulus between 3 and 7 pixels from the target centroid. We were unable to apply the highresolution gain map of the sweet spot pixel because WISE $\mathrm{J} 1405+5534$ was on average 0.25 pixels away from the spot and the map is only $0.5 \times 0.5$ pixel in size. Furthermore, we did not apply the pixel phase correction using the IDL routine pixel_phase_correct_gauss provided by the Spitzer Science Center because it did not reduce the scatter in the data. An alternative method to correct for the pixel phase effect used by Knutson et al. (2008) and Heinze et al. (2013) was also tested, but it too did not reduce the scatter in the data. From these tests, we can conclude that the pixel phase corrections are not significant above the random noise in our data, probably due to the faintness of our target. Finally, data points that are clear outliers (i.e., the total number of electrons exceeded the median intensity level by more than 50 times the median absolute deviation ${ }^{9}$ ) were removed; this resulted in the removal of 8 and 3 points in the epoch 1 [3.6] and [4.5] light curves,

\footnotetext{
9 For a data set $\boldsymbol{X}=\left\{x_{1}, x_{2}, \ldots, x_{N}\right\}$, the median absolute deviation (MAD) is given by MAD $=\operatorname{median}\left(\left|x_{i}-\operatorname{median}(\boldsymbol{X})\right|\right)$.
}

respectively, and 10 and 3 points in the epoch 2 [3.6] and [4.5] light curves, respectively.

The final time series in both epochs after dividing by the median intensity level is shown in Figure 1. The scatter in the [3.6] data is larger than that in the [4.5] data because WISE $\mathrm{J} 1405+5534$ is fainter by a factor of 7 at $3.6 \mu \mathrm{m}$ due to the strong $\nu_{3}$ fundamental band of $\mathrm{CH}_{4}$ centered at $3.3 \mu \mathrm{m}$.

\section{ANALYSIS}

\subsection{Characterizing the Variability}

Visual inspection of Figure 1 shows that WISE J1405+5534 is variable, with semi-amplitudes of a few percent at both [3.6] and [4.5]. This is the first detection of variability in a spectroscopically confirmed Y dwarf and suggests that the mid-infrared variability observed in late-type $\mathrm{T}$ dwarfs by Metchev et al. (2015) continues across the $\mathrm{T} / \mathrm{Y}$ boundary. There are, however, obvious differences in the light curves between the two epochs, including the lack of clear variability at [3.6] in epoch 1, the near-sinusodial shape of the [3.6] and [4.5] epoch 2 light curves, and the more complex shape (i.e., not purely sinusoidal) of the [4.5] epoch 1 light curve. These differences indicate that the underlying cause or causes of the observed variability in WISE J1405+5534 evolve on timescales of months. Finally, although clear semi-periodic or periodic variability is detected at the few percent level in the relative light curves, the average [3.6]-[4.5] color shows no change between the two epochs because the average flux levels at [3.6] and [4.5] are equal within the (Poisson) uncertainties.

In order to measure the amplitude, phase, and period of the roughly sinusoidal epoch 2 light curves, we assume the data are generated from the following probabilistic model:

$$
D_{i}=C+A \sin \left(\frac{2 \pi}{P} t_{i}+\phi\right)+\epsilon,
$$

where $D_{i}$ is a random variable for the number of electrons detected at the $i$ th time $t_{i}, C$ is an additive constant, $A$ is the semi-amplitude, $P$ is the period, $\phi$ is the phase, and $\epsilon$ is a random variable that accounts for a measurement error that has a mean of zero and a variance of $\sigma^{2}$. We can determine the joint probability distribution function of these five parameters given our $N$ observations (denoted as $\boldsymbol{d}=\left\{d_{1}, d_{2}, d_{3}, \cdots, d_{N}\right\}$ ) using Bayes' Theorem

$$
p(C, A, P, \phi, \sigma \mid \boldsymbol{d}) \propto \mathcal{L}(\boldsymbol{d} \mid C, A, P, \phi, \sigma) p(C, A, P, \phi, \sigma),
$$

where $p(C, A, P, \phi, \sigma \mid d)$ is the posterior distribution, $\mathcal{L}(\boldsymbol{d} \mid C, A, P, \phi, \sigma)$ is the likelihood, and $p(C, A, P, \phi, \sigma)$ is the prior distribution. We assume that the random variable $\epsilon$ follows a normal distribution, thus, assuming the data points are independent, the likelihood is given by

$$
\begin{aligned}
& \mathcal{L}(\boldsymbol{d} \mid C, A, P, \phi, \sigma)=\left(\frac{1}{\sqrt{2 \pi \sigma^{2}}}\right)^{N} \\
& \quad \times \exp \left[-\sum_{i=0}^{N}\left(\frac{\left[d_{i}-C-A \sin \left(2 \pi t_{i} / P+\phi\right)\right]^{2}}{2 \sigma^{2}}\right)\right] .
\end{aligned}
$$

Visual inspection of Figure 1 clearly shows outliers in the data. We model the entire time series following the procedure described in Hogg et al. (2010), assuming that the good data 

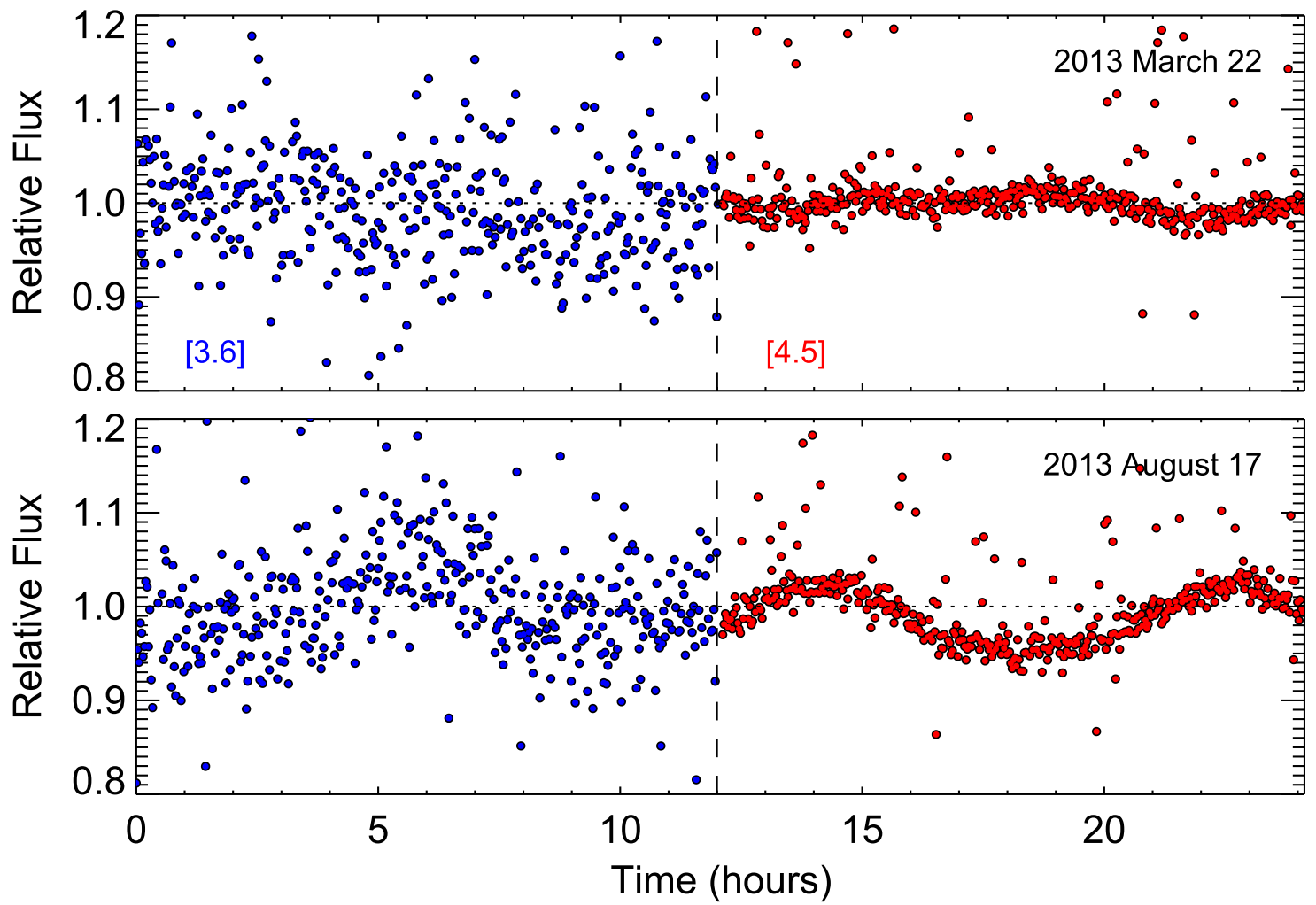

Figure 1. Normalized IRAC [3.6] and [4.5] photometry on 2013 March 22 (top) and 2013 August 17 (bottom) plotted from the beginning of [3.6] observations in each epoch on the same timescale. The ordinate range was selected to emphasize the variability in the light curves and thus some outlier data points with relative fluxes outside of this range are not shown. The scatter in the [3.6] data is larger than that in the [4.5] data because WISE J1405+5534 is fainter by a factor of 7 at $3.6 \mu \mathrm{m}$ due to the strong $\nu_{3}$ fundamental band of $\mathrm{CH}_{4}$ centered at $3.3 \mu \mathrm{m}$. The data used to create this figure are available.

points are generated from Equation (1) and the outliers (i.e., "bad" data points) are generated from a normal distribution with a mean of $Y_{\mathrm{bad}}$ and a variance of $\sigma_{\mathrm{bad}}^{2}$. The likelihood function then becomes

$$
\begin{aligned}
\mathcal{L}\left(\boldsymbol{d} \mid P_{\mathrm{b}}, Y_{\mathrm{bad}}, \sigma_{\mathrm{bad}}, C, A, P, \phi, \sigma\right) & \\
= & \prod_{i=0}^{N}\left[\frac{1-P_{\mathrm{bad}}}{\sigma \sqrt{2 \pi}} \exp \left(-\frac{\left[d_{i}-C-A \sin \left(2 \pi t_{i} / P+\phi\right)\right]^{2}}{2 \sigma^{2}}\right)\right. \\
& \left.+\frac{P_{\mathrm{bad}}}{\sqrt{2 \pi \sigma_{\mathrm{bad}}^{2}}} \exp \left(-\frac{\left[d_{i}-Y_{\mathrm{bad}}\right]^{2}}{2 \sigma_{\mathrm{bad}}^{2}}\right)\right],
\end{aligned}
$$

where $P_{\mathrm{b}}$ is the probability that a data point is bad (see Hogg et al. for a derivation of this equation).

We assume that the joint prior distribution can be factored as the product of individual probability distribution functions and use uniform priors for all parameters (see Table 1). We sampled the joint posterior distribution using a Markov Chain Monte Carlo method employed by the emcee package (ForemanMackey et al. 2013). We used 1000 walkers in the eightdimensional parameter space to model the light curves and kept 700,000 samples after discarding an initial burn-in sample. With the joint posterior distribution in hand, we computed posterior distributions for each of the eight model parameters by marginalizing over the other seven parameters. The values corresponding to the 16th, 50th, and 84th percentiles of the marginalized distributions are given in Table 1 and the resulting best-fit models and residuals $(O-C)$ are shown in Figure 2.

The semi-amplitudes, periods, and phases of the [3.6] and [4.5] epoch 2 light curves are equal within the uncertainties. Since the [4.5] period has a smaller uncertainty, we identify the rotation period of WISE J1405+5534 based on the [4.5] data as $8.54 \pm 0.08 \mathrm{hr}$. The uncertainties at [3.6] and [4.5] of 0.045 and 0.011 (45 and $11 \mathrm{mmag}$ ) are factors of $\sim 3$ and $\sim 2$ larger than the theoretical photon limit. The residuals also show no largescale trends, which suggests that a simple sine curve is an accurate representation of the data.

\subsection{Interpreting the Variability}

There are several mechanisms that can induce variability in the integrated light of low-mass stars and brown dwarfs, including magnetic activity, non-uniform surface opacities, either in the form of chemical abundance variations or heterogeneous cloud coverage, and non-uniform temperature profiles resulting in "hot" and/or "cold" spots. Magnetic activity is often ignored in the study of cool brown dwarfs because the atmospheres of brown dwarfs are predominantly neutral and thus the magnetic field lines presumably have a difficult time coupling to the gas (e.g., Gelino et al. 2002; Mohanty et al. 2002). The degree to which non-uniform surface opacities and non-uniform temperature profiles contribute to the observed levels of variability in $\mathrm{L}$ and $\mathrm{T}$ dwarfs and the timescales on which they operate are still an open question, although some progress has been made. 

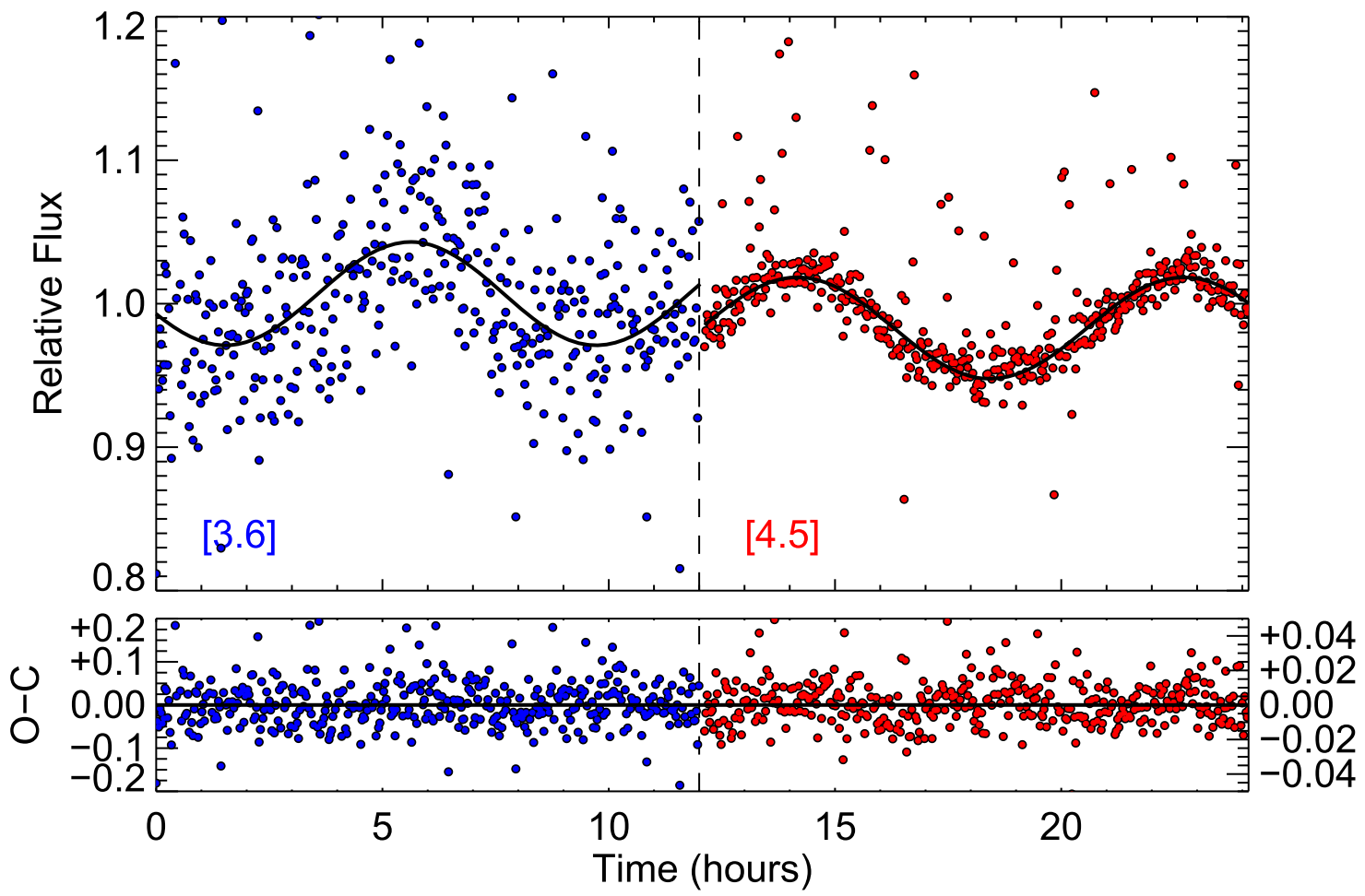

Figure 2. Top: best-fitting sine curve overplotted on the epoch 2 [3.6] and [4.5] data. Bottom: the residuals $(O-C)$ show no large-scale structure, indicating a sine curve is a reasonable model of the data.

\subsubsection{Vertical Extent of Spot(s)}

Before comparing our observations to the detailed predictions of model atmospheres, we first discuss what can be learned about the underlying cause of the variability by exploiting the near-simultaneous, multi-wavelength nature of the observations. The emergent spectra of brown dwarfs are distinctly non-Planckian and as a result, different wavelengths probe different layers of the atmosphere. Detecting and characterizing variability at multiple wavelengths simultaneously can therefore inform our understanding of the vertical extent of the underlying cause (or causes) of the variability. As an example, the top panel of Figure 3 shows the emergent intensity for three atmospheric models from Morley et al. (2014a) with $T_{\text {eff }}=400 \mathrm{~K}, \log g=4.5\left(\mathrm{~cm} \mathrm{~s}^{-2}\right), f_{\text {sed }}=3$ and $^{10}$ cloud coverage fractions of $0 \%, 50 \%$, and $100 \%(h=1,0.5,0$, see Section 3.2.3 and Equation (8)). The lower panel of Figure 3 shows the brightness temperature of the same three models. The brightness temperature is the temperature of a blackbody that has the same intensity as the model at a given wavelength. Since temperature typically increases with increasing depth into an atmosphere, the brightness temperature can be used as a proxy for depth in the atmosphere. Also given are the atmospheric pressures derived using the model pressuretemperature profile that corresponds to the brightness temperatures.

The red-optical $(0.70-1.0 \mu \mathrm{m})$ through mid-infrared wavelengths probe a large range of atmospheric temperatures and pressures. In general, the [3.6] and [4.5] IRAC bands probe

\footnotetext{
10 The sedimentation efficiency parameter describes the efficiency of cloud particle sedimentation relative to turbulent mixing (Ackerman \& Marley 2001). Larger values of $f_{\text {sed }}$ imply larger particle sizes and geometrically thinner clouds. Cloudless models are denoted as $f_{\text {sed }}=\mathrm{nc}$, where nc means "no clouds."
}

Table 1

Epoch 2 Sine Curve Model Parameters

\begin{tabular}{llcc}
\hline \hline Model Parameter & Prior $^{\mathrm{a}}$ & {$[3.6]$ Value $^{\mathrm{b}}$} & [4.5] Value \\
\hline Constant $C$ & $\mathcal{U}(0.9,1.1)$ & $1.007 \pm 0.003$ & $0.9834 \pm 0.0006$ \\
Amplitude $A(\%)$ & $\mathcal{U}(0,5)$ & $3.6 \pm 0.4$ & $3.54 \pm 0.09$ \\
Period $P$ (hours) & $\mathcal{U}(7,10)$ & $8.2 \pm 0.3$ & $8.54 \pm 0.08$ \\
Phase $\phi$ (degrees) & $\mathcal{U}(0,360)$ & $203_{-11}^{+12}$ & $213 \pm 7$ \\
Standard deviation $\sigma$ & $\mathcal{U}(0,0.5)$ & $0.045 \pm 0.002$ & $0.0109 \pm 0.0005$ \\
Bad data mean $Y_{\text {bad }}$ & $\mathcal{U}(0.5,2.5)$ & $1.44_{-0.08}^{+0.09}$ & $1.07 \pm 0.02$ \\
Bad data standard devia- & $\mathcal{U}(0,1)$ & $0.50_{-0.05}^{+0.6}$ & $0.11 \pm 0.01$ \\
$\quad$ tion $\sigma_{\text {bad }}$ & & & \\
Bad data probability $P_{\text {bad }}$ & $\mathcal{U}(0,0.25)$ & $0.10 \pm 0.02$ & $0.12 \pm 0.02$ \\
\hline
\end{tabular}

Notes.

a $\mathcal{U}(a, b)$ denotes a uniform distribution over the range $a$ to $b$.

$\mathrm{b}$ The values reported correspond to the 16th, 50th, and 84th percentiles of the marginalized posterior distribution.

higher atmospheric layers (i.e., lower temperatures, lower pressures) than near-infrared wavelengths $(1-2.5 \mu \mathrm{m})$. However, due to the strong methane absorption band centered at $3.3 \mu \mathrm{m}$, the [3.6] band probes atmospheric layers with $P \approx 0.8$ bar and $T \approx 400 \mathrm{~K}$, while the [4.5] band probes layers with $P \approx 3$ bar and $T \approx 600 \mathrm{~K}$. Although the brightness temperature is a useful proxy for the pressure level at which radiation emerges at a given wavelength, multiple layers of the atmosphere actually contribute to the emergent flux at each wavelength. In addition, the Spitzer bandpasses are not delta functions and thus the radiation detected through them actually emerges from an even broader range of pressure levels.

A more accurate representation of what pressure levels contribute thermal emission to the emergent flux at a given wavelength is given by the contribution function (Chamberlain 

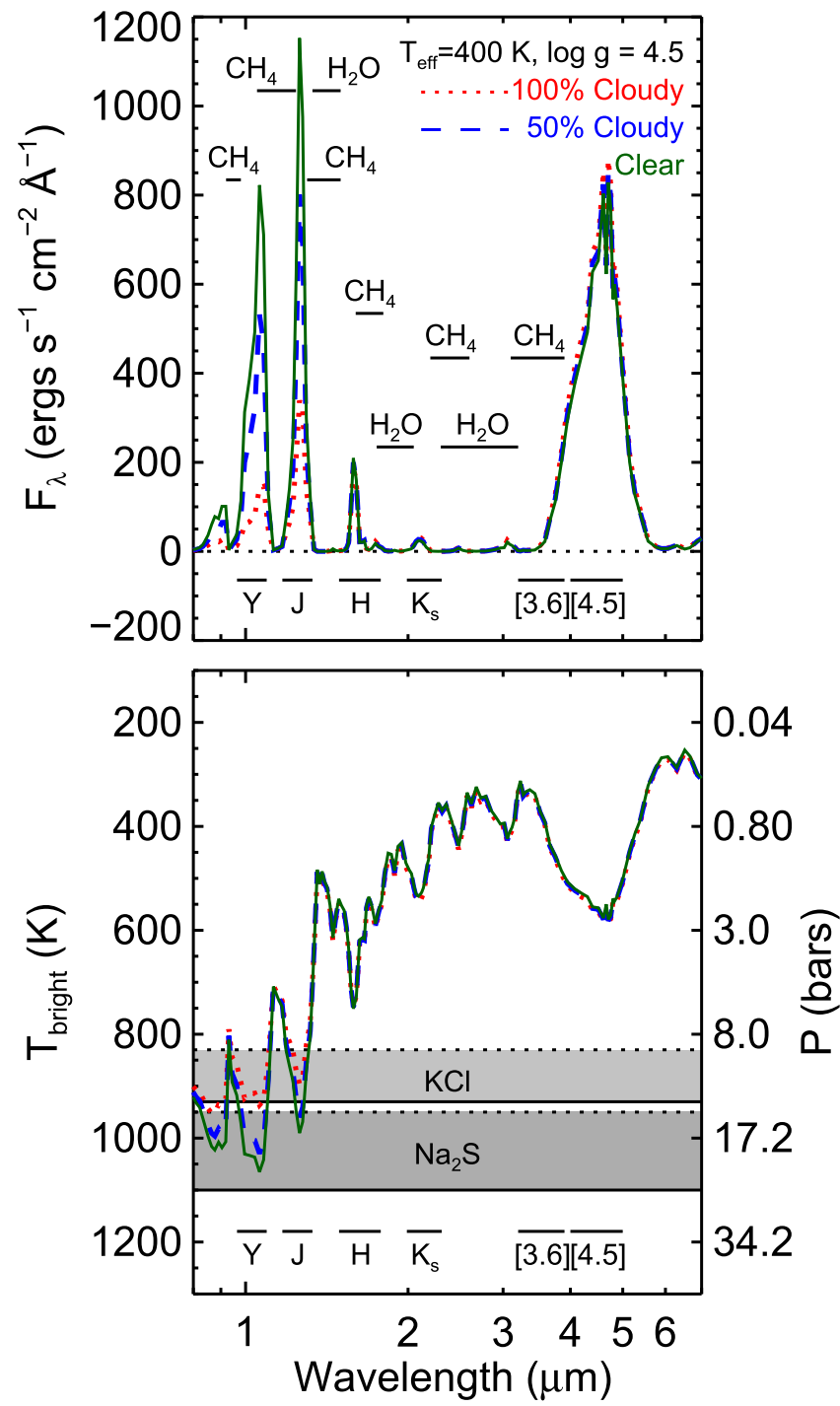

Figure 3. Top: solar metallicity partly cloudy model spectra from Morley et al. (2014a) at $T_{\text {eff }}=400 \mathrm{~K}, \log g=4.5\left(\mathrm{~cm} \mathrm{~s}^{-2}\right), f_{\text {sed }}=3$ for three different cloud coverages, $100 \%$ cloudy, 50\% cloud, and clear. Prominent molecular absorption bands are identified along with the wavelength range between the half-power points of the $Y$ band (Hillenbrand et al. 2002), the 2MASS JHK bands (Cohen et al. 2003), and the IRAC [3.6] and [4.5] bandpasses (Fazio et al. 2004). Bottom: brightness temperatures for the same models. The locations of the $\mathrm{Na}_{2} \mathrm{~S}$ and $\mathrm{KCl}$ cloud decks are indicated. The pressure corresponding to the brightness temperature derived using the $\mathrm{P} / \mathrm{T}$ profile of the clear model is also given.

\section{\& Hunten 1987)}

$$
\operatorname{cf}(\lambda, P)=B_{\nu}(\lambda, T) e^{-\tau_{\lambda}} \frac{d \tau_{\lambda}}{d \log P},
$$

where $B_{\nu}(\lambda, T)$ is the Planck function, $P$ is the atmospheric pressure, and $\tau_{\lambda}$ is the vertical monochromatic optical depth; the integral of the contribution function over pressure in a semiinfinite atmosphere gives the specific intensity at the top of the atmosphere:

$$
I_{\lambda}(\lambda, P=0)=\int_{\infty}^{0} \operatorname{cf}(\lambda, P) d \log P .
$$

Since the [3.6] and [4.5] bandpasses have a finite width, we must compute a band-averaged contribution function, which is given by the integral of the contribution function over the system response function $S(\lambda)$,

$$
\langle\operatorname{cf}(P)\rangle=\frac{\int_{0}^{\infty} \operatorname{cf}(\lambda, P) S(\lambda) d \lambda}{\int_{0}^{\infty} S(\lambda) d \lambda},
$$

where the integral over the band-averaged contribution function is now proportional to flux detected through the bandpass.

We computed contribution functions at two thousand wavelengths and sixty pressure levels within a cloudless model atmosphere with $T_{\text {eff }}=400 \mathrm{~K}, \log g=4.5\left(\mathrm{~cm} \mathrm{~s}^{-2}\right)$ and then computed the band-averaged contribution functions for the $J$, $H$, [3.6], and [4.5] bands using the full array average system response functions of the [3.6] and [4.5] bands ${ }^{11}$ and the Two Micron All Sky Survey (2MASS) $J$ and $H$-band transmission curves from Cohen et al. (2003). Figure 4 shows the resulting normalized band-averaged contribution functions for the $J, H$, [3.6] and [4.5] bandpasses along with the temperature-pressure profile for the cloudless $T_{\text {eff }}=400 \mathrm{~K}, \log g=4.5\left(\mathrm{~cm} \mathrm{~s}^{-2}\right)$ model.

Although on average the [3.6] band probes slightly higher pressures than the [4.5] band, there is considerable overlap and thus much of the emergent flux in the Spitzer bandpasses comes from the same layers of the atmosphere. Nevertheless, a few general conclusions can be still drawn. If the variability is caused by temperature perturbations at depth, then the fact that the epoch 2 [3.6] and [4.5] light curves have the same phase within the uncertainties suggests that whatever phenomenon causes these perturbations extends over at least these atmospheric layers, e.g., 3-0.1 bars, although the details of the vertical variation and the underlying mechanism must be further addressed by future observations and analysis. In contrast, the lack of variability detected at [3.6] in the epoch 1 data suggests that the phenomenon is either absent from the upper atmosphere $\lesssim 0.6$ bar or has weakened in strength such that its effect on the emergent flux falls below our detection threshold. The differences in shape of the two [4.5] light curves also suggest that changes in the phenomenon deeper in the atmosphere have also occurred between the two epochs. If the observed variability is caused by temperature variations, then our observations provide the first evidence for the evolution of weather patterns with depth on the timescales of months for $\mathrm{Y}$ dwarfs.

\subsubsection{Geometric Spot Model}

Before delving into the possible underlying cause or causes of the observed variability, we first model the variability using a simple geometric spot model because it requires the least number of assumptions. The simplicity and similarity of the WISE J1405+5534 epoch 2 light curves suggests that a single feature may explain the variability. We therefore modeled both the [3.6] and [4.5] epoch 2 light curves with a single, bright circular spot using the equations of Dorren (1987). The brown dwarf is characterized by a rotation period, the inclination to the line of sight, and the brightness of the spot-free photosphere at [3.6] and [4.5]. The spot is characterized by a size (radius, in

\footnotetext{
11 Files 080924ch1trans_full.txt and 080924ch2trans_full. txt obtained on 2014 May 22 from http://irsa.ipac.caltech.edu/data/ SPITZER/docs/irac/calibrationfiles/spectralresponse/.
} 

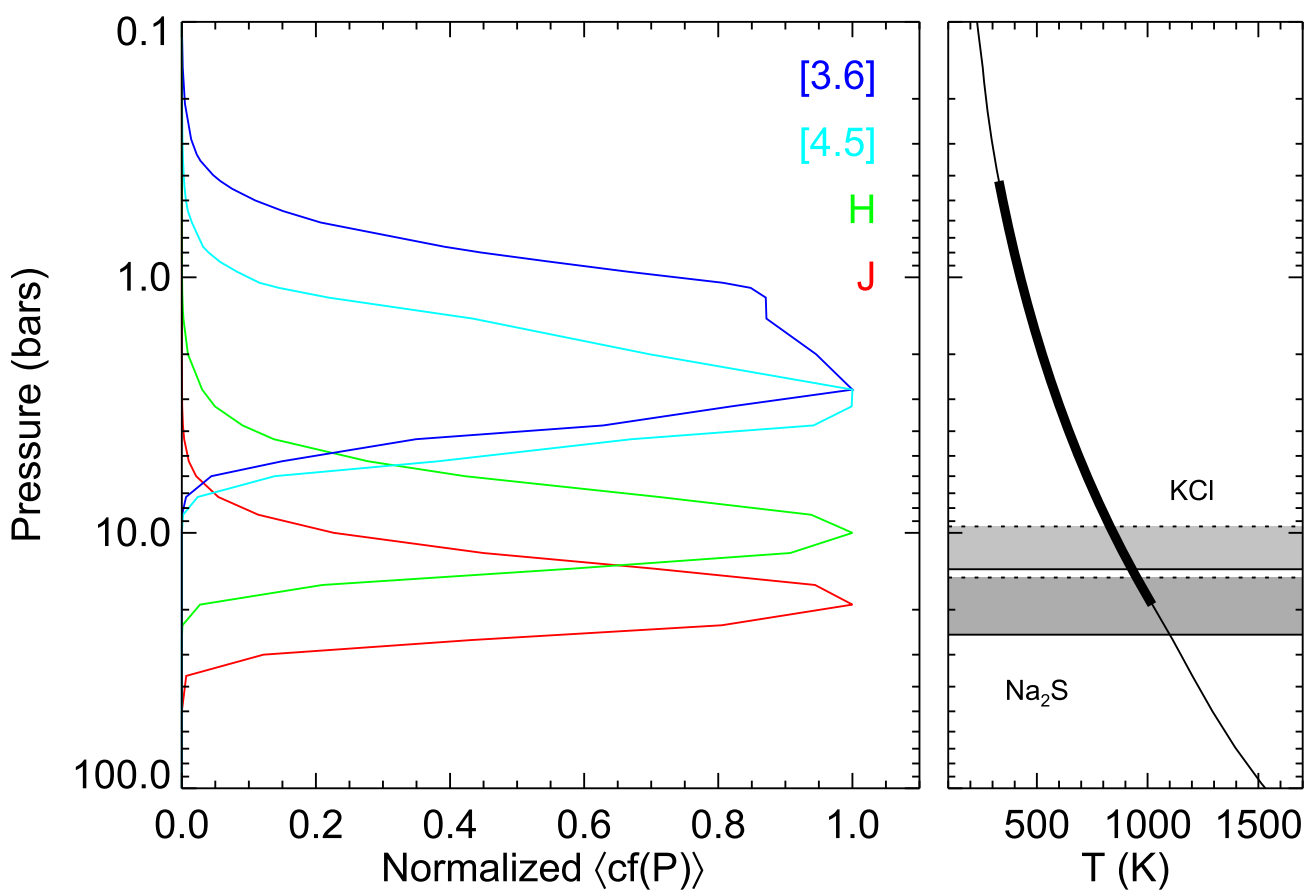

Figure 4. Left: band-averaged contribution functions normalized to unity at their peak for the $J, H$, and [3.6] and [4.5] bandpasses for a cloudless model atmosphere with $T_{\text {eff }}=400 \mathrm{~K}$ and $\log g=4.5\left(\mathrm{~cm} \mathrm{~s}^{-2}\right)$. Right: the temperature/pressure profile for the same model with the location of the $\mathrm{KCl}$ and $\mathrm{Na}_{2} \mathrm{~S}$ clouds indicated. The thick line denotes the region of the atmosphere that is convective.

radians), a position (longitude and latitude), and the spot-tophotosphere brightness ratio at [3.6] and [4.5]. This ratio is defined as the flux per unit area of the spot divided by the flux per unit area of the unspotted photosphere; it is greater than one for bright spots. Lacking constraints on the limb darkening, we adopt linear limb darkening coefficients of 0.5 for both the photosphere and spot $\left(\mu_{*}=\mu_{s}=0.5\right.$ in Dorren's notation.)

To explore the possible values of the parameters, we use the emcee code (Foreman-Mackey et al. 2013) to perform a Markov Chain Monte Carlo calculation of the joint posterior distribution using the same model for bad data that is described in Section 3.1. We use 1000 walkers in the seventeendimensional parameter space (11 parameters for the spot model and 6 parameters to account for the bad data) to model the light curves and kept 2.9 million samples after discarding an initial burn-in sample. The values corresponding to the 16th, 50th, and 84th percentiles of the marginalized distributions of each parameter are given in Table 2. We find that the rotation period is $8.50 \pm 0.05 \mathrm{hr}$ (consistent with the period derived Section 3.1), the size of the spot is $14^{\circ} \pm 3^{\circ}$, and the spotto-photosphere brightness ratio at [3.6] is $4.0_{-0.9}^{+1.2}$ and at [4.5] is $3.8_{-0.8}^{+1.0}$. Although a broad range of spot brightnesses are consistent with the data, the brightness in the two bands must be similar since the [3.6]/[4.5] ratio is $1.05 \pm 0.08$. A ratio of near unity is also consistent with the fact that the [3.6] and [4.5] band-averaged contribution functions overlap (see Section 3.2.1), which suggests that a single spot could account for the variability in both bands. The light curves corresponding to the best-fit values and residuals $(O-C)$ are shown in Figure 5.

\subsubsection{Partly Cloudy Model Atmospheres}

We now compare our observations to the predictions of the one-dimensional model atmospheres of Morley et al. (2014a).
Table 2

Epoch 2 Spot Model Parameters

\begin{tabular}{|c|c|c|}
\hline Model Parameter & Prior $^{\mathrm{a}}$ & Value $^{b}$ \\
\hline Spot radius $\alpha$ (degrees) & $\mathcal{U}(0,90)$ & $14 \pm 3$ \\
\hline Spot latitude $\chi$ (degrees) & $\mathcal{U}(0,90)$ & $13 \pm 4$ \\
\hline Stellar inclination $i$ (degrees) & $\mathcal{U}(0,90)$ & $16_{-4}^{+5}$ \\
\hline Spot longitude $\psi$ (degrees) & $\mathcal{U}(0,360)$ & $99 \pm 7$ \\
\hline Period $P$ (hours) & $\mathcal{U}(0,19)$ & $8.50 \pm 0.05$ \\
\hline [3.6] Photosphere flux $F_{*,[3.6]}$ & $\mathcal{U}(0.5,15)$ & $0.972_{-0.007}^{+0.005}$ \\
\hline $\begin{array}{l}\text { [3.6] Spot-to-photosphere flux } \\
\text { ratio }\left(F_{s} / F_{*}\right)_{[3.6]}\end{array}$ & $\mathcal{U}(1,99)$ & $4.0_{-0.9}^{+1.2}$ \\
\hline [4.5] Photosphere flux $F_{*,[4.5]}$ & $\mathcal{U}(0.5,15)$ & $0.951_{-0.005}^{+0.003}$ \\
\hline $\begin{array}{l}\text { [4.5] Spot-to-photosphere flux } \\
\text { ratio }\left(F_{s} / F_{*}\right)_{[4.5]}\end{array}$ & $\mathcal{U}(1,99)$ & $3.8_{-0.8}^{+1.0}$ \\
\hline [3.6] Standard deviation $\sigma_{[3.6]}$ & $\mathcal{U}(0,0.5)$ & $0.044 \pm 0.002$ \\
\hline [4.5] Standard deviation $\sigma_{[4.5]}$ & $\mathcal{U}(0,0.5)$ & $0.0102 \pm 0.0005$ \\
\hline [3.6] Bad data mean $Y_{[3.6], \text { bad }}$ & $\mathcal{U}(0.5,2.5)$ & $1.4_{-0.06}^{+0.07}$ \\
\hline [3.6] Bad data standard deviation $\sigma_{[3.6] \text {,bad }}$ & $\mathcal{U}(0,1)$ & $0.48_{-0.04}^{+0.05}$ \\
\hline [3.6] Bad data probability $P_{[3.6] \text {,bad }}$ & $\mathcal{U}(0,0.25)$ & $0.10 \pm 0.02$ \\
\hline [4.5] Bad data mean $Y_{[4.5], \text { bad }}$ & $\mathcal{U}(0.5,2.5)$ & $1.07 \pm 0.01$ \\
\hline [4.5] Bad data standard deviation $\sigma_{[4.5], \text { bad }}$ & $\mathcal{U}(0,1)$ & $0.10 \pm 0.01$ \\
\hline [4.5] Bad data probability $P_{[4.5] \text {,bad }}$ & $\mathcal{U}(0,0.25)$ & $0.13 \pm 0.02$ \\
\hline
\end{tabular}

Notes.

${ }^{\text {a }} \mathcal{U}(a, b)$ denotes a uniform distribution over the range $a$ to $b$.

$\mathrm{b}$ The values reported correspond to the 16th, 50th, and 84th percentiles of the marginalized posterior distribution.

These models are computed assuming solar abundance and include salt $(\mathrm{KCl})$, sulfide $\left(\mathrm{MnS}, \mathrm{Na}_{2} \mathrm{~S}\right.$, and $\left.\mathrm{ZnS}\right)$, chromium, and water ice clouds (Morley et al. 2012, 2014b). The model atmospheres are static and thus show no variation in intensity with time. We can, however, compare the measured semi- 

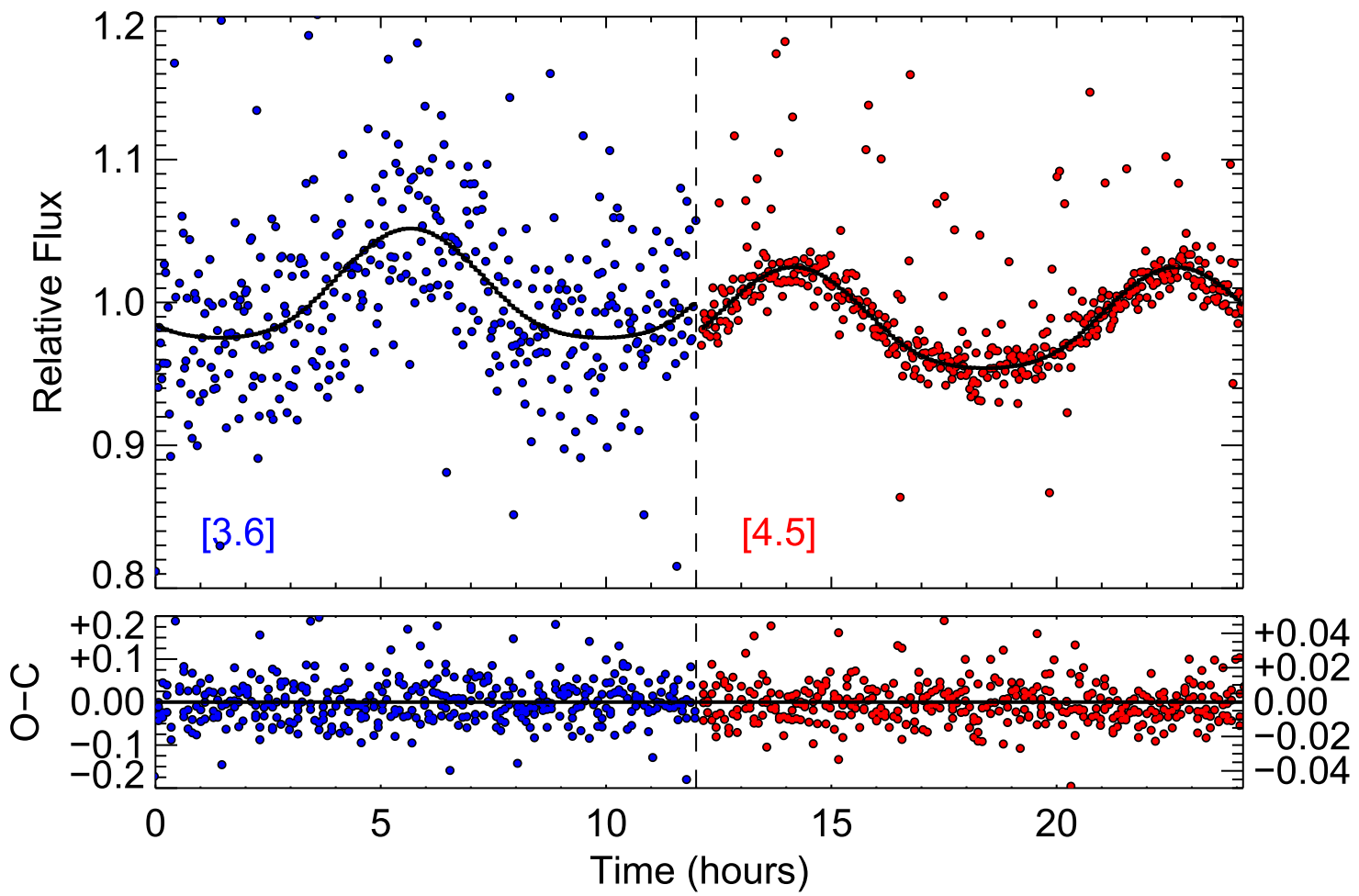

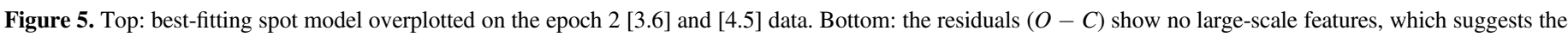
single spot model is a reasonable model of the data.

amplitudes of the WISE J1405+5534 epoch 2 light curves to those computed from models with different cloud coverage fractions. Partly cloudy atmospheres are modeled following the prescription of Marley et al. (2010), in which the flux through a cloudy column of gas and a clear column of gas are computed separately and then combined to calculate the total flux through a partly cloudy column as

$$
F_{\text {total }}=h F_{\text {clear }}+(1-h) F_{\text {cloudy }},
$$

where $h$ is a parameter that ranges from 0 to 1 and gives the fraction of the atmosphere covered by holes. In order to simulate all possible variations in $h$ from 0 to 1 , a suite of 66 models with $T_{\text {eff }}=400,500 \mathrm{~K}, \log g=4.0,4.5,5.0\left(\mathrm{~cm} \mathrm{~s}^{-2}\right)$, $f_{\text {sed }}=3$, and $h$ values ranging from 0 to 1 in steps of 0.1 was generated. These models cover the range of effective temperatures and surface gravities $\left(T_{\text {eff }}=370-470 \mathrm{~K}\right.$, $\left.\log g=4.12-4.78\left(\mathrm{~cm} \mathrm{~s}^{-2}\right)\right)$ computed by Dupuy \& Kraus (2013) for WISE J1405+5534. The effective temperature and surface gravity values from Dupuy \& Kraus (2013) were computed by first applying a model-derived bolometric correction to observed absolute magnitudes of WISE J1405 +5534 and then comparing the resulting bolometric luminosities to evolutionary models at ages of 1 and 5 Gyr. It should be noted that any conclusions we draw from these comparisons must be tempered against the fact that these models do not fit the near-infrared to mid-infrared spectral energy distributions of the cold late-type $\mathrm{T}$ and $\mathrm{Y}$ dwarfs well (Schneider et al. 2015).

Perhaps the simplest way to simulate brown dwarf variability using these models is to assume that one hemisphere has an $h$ value of $h_{1}$ and the other hemisphere has an $h$ value of $h_{2}$. The rotation of the brown dwarf would then modulate the total intensity and produce a measurable difference in the integrated intensity. For a given effective temperature and surface gravity, we computed synthetic semi-amplitudes for all possible $\left(h_{1}, h_{2}\right)$ pairs by computing the average flux densities through the Spitzer [3.6] and [4.5] following the prescription of Cushing et al. (2006), then computing the semi-amplitude as

$$
A_{\lambda}=\frac{\max \left[f_{\nu, h_{1}}(\lambda), f_{\nu, h_{2}}(\lambda)\right]}{\operatorname{average}\left[f_{\nu, h_{1}}(\lambda), f_{\nu, h_{2}}(\lambda)\right]}-1 .
$$

Figure 6 shows the results for effective temperatures of 400 and $500 \mathrm{~K}$ and $\log$ surface gravities of 4.0 and $5.0\left(\mathrm{~cm} \mathrm{~s}^{-2}\right)$. Several important conclusions can be drawn from this figure. First, the models predict that the semi-amplitudes at both wavelengths are larger at $T_{\text {eff }}=500 \mathrm{~K}$. Second, variations in surface gravity at a fixed effective temperature have a small $(\lesssim 1 \%)$ effect on the semi-amplitudes. And finally, for any given $\left(h_{1}, h_{2}\right)$ pair, the models always predict a larger semiamplitude at [3.6]. Also shown in each panel as solid lines are the $3 \sigma$ contours corresponding to the observed epoch 2 semiamplitudes; the $3 \sigma$ contours for the other wavelength are shown as dotted lines. Agreement between the models and observations occurs if the two contours overlap, since a single $\left(h_{1}, h_{2}\right)$ can account for the observed semi-amplitudes at both wavelengths. While there is no overlap in any of the panels, the two contours come close at $T_{\text {eff }}=500 \mathrm{~K}$, which, given the complexity of the model atmospheres, suggests that patchy clouds are a plausible mechanism for explaining the variability observed for WISE J1405+5534 at [3.6] and [4.5]. It should be noted, however, that a hypothetical two-hemisphere brown dwarf constructed using models with different $h$ values is unphysical since the pressure-temperature profiles of the two models do not converge to the same adiabat at depth due to the differences in cloud opacity. In effect, we are comparing the 

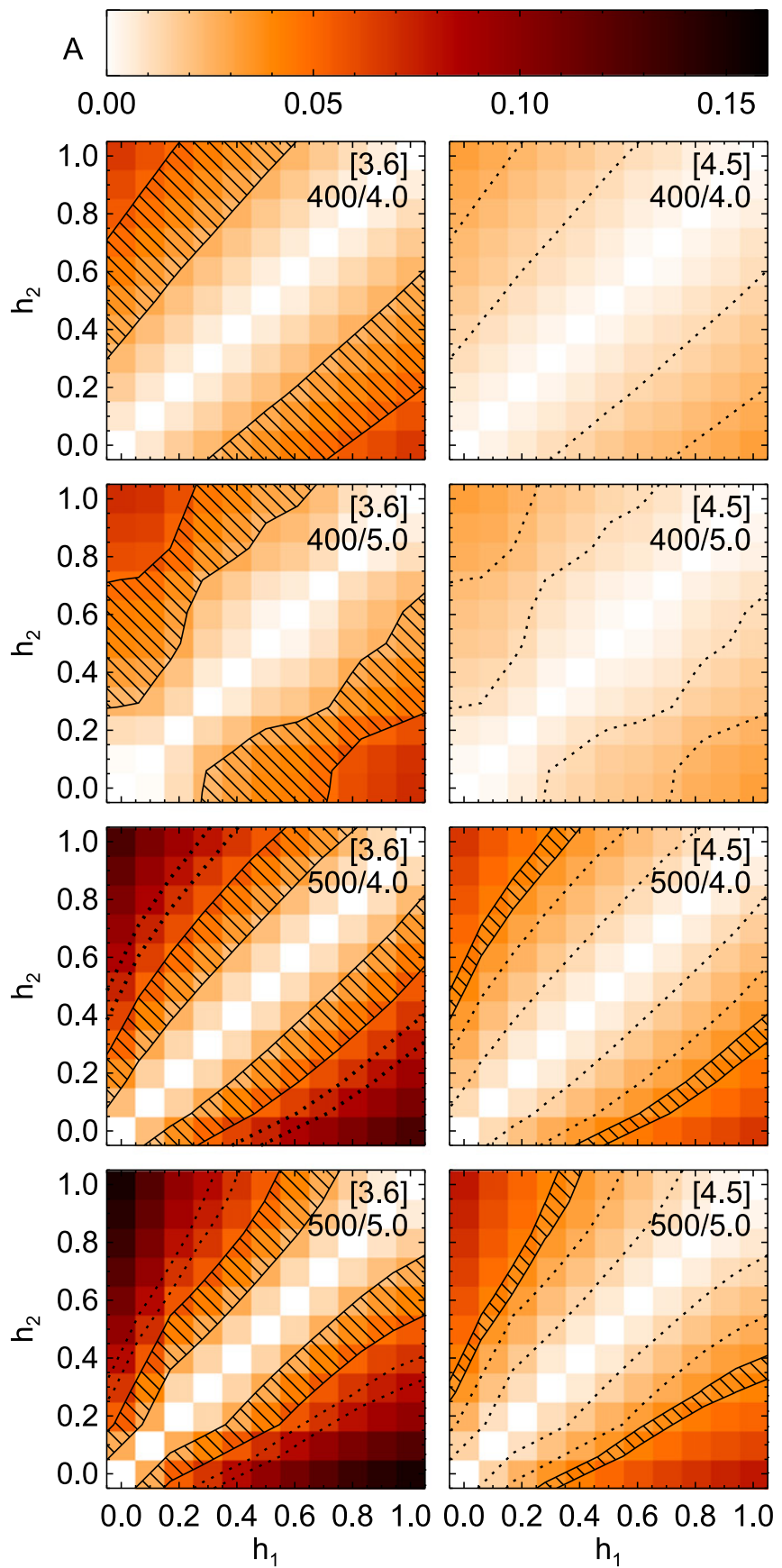

Figure 6. Semi-amplitudes at [3.6] (left column) and [4.5] (right column) arising from changes in the cloudiness fraction $\left(h_{1} \rightarrow h_{2}\right)$ at different effective temperatures and surface gravities $\left(T_{\text {eff }} / \log g\right)$. Since $h_{1} \rightarrow h_{2}$ is equivalent to $h_{2} \rightarrow h_{1}$, each panel is symmetric across the diagonal. In each panel, the $\pm 3 \sigma$ range of the measured semi-amplitudes of the second-epoch WISE J1405 +5534 light curve are shown as hashed regions, while $\pm 3 \sigma$ ranges for the other wavelength are encompassed by the dotted lines. The model [4.5] semiamplitudes for the $400 \mathrm{~K}$ models fall below the observational level and thus no regions are shown. Since the contours never overlap, there are no pairs of $h$ values that simultaneously reproduce the [3.6] and [4.5] observations.

atmospheres of two different brown dwarfs rather than the two hemispheres of a single brown dwarf.

An alternative method that is self-consistent is described by Morley et al. (2014a), who describe a model with a single $h$ value (e.g., $h=0.5$ ) that is used to generate two model spectra with different $h$ values (e.g., 0.4 and 0.6). The possible

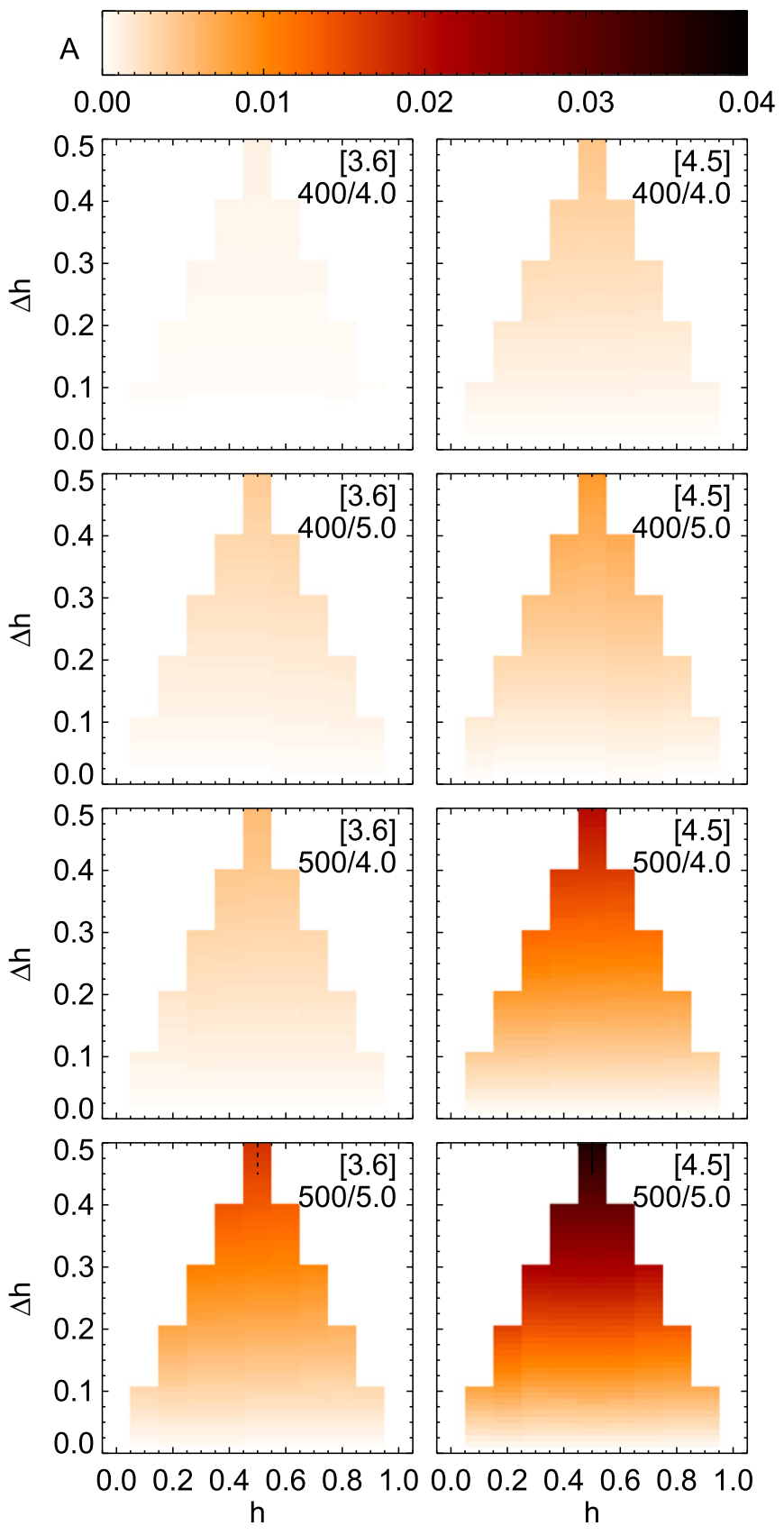

Figure 7. Semi-amplitudes at [3.6] (left column) and [4.5] (right column) arising from changes in the cloudiness fraction $\Delta h$ for a fixed $h$ value at different effective temperatures and surface gravities $\left(T_{\text {eff }} / \log g\right)$. With the exception of the $T_{\text {eff }}=500 \mathrm{~K}, \log g=5.0\left(\mathrm{~cm} \mathrm{~s}^{-2}\right), h=0.5, \Delta h=0.5 \mathrm{semi}-$ amplitude, the models do not predict semi-amplitudes high enough to match the observations and thus regions similar to those shown in Figure 6 are not indicated.

combinations of $h$ values are limited, however, because the two values must always average to the $h$ value of the original model so that on the whole, the atmosphere is self-consistent. To compute model spectra with $h$ values given by $h_{0}-\Delta h$ and $h_{0}+\Delta h$, we use the clear and cloudy columns for a model with an $h=h_{0}$ and Equation (8). Synthetic semi-amplitudes were computed as described above and the results are shown in Figure 7. Just like the previous method, the semi-amplitudes are larger at $T_{\text {eff }}=500 \mathrm{~K}$ but are much lower $(<5 \%)$ than those resulting from simply varying the $h$ values as described above 


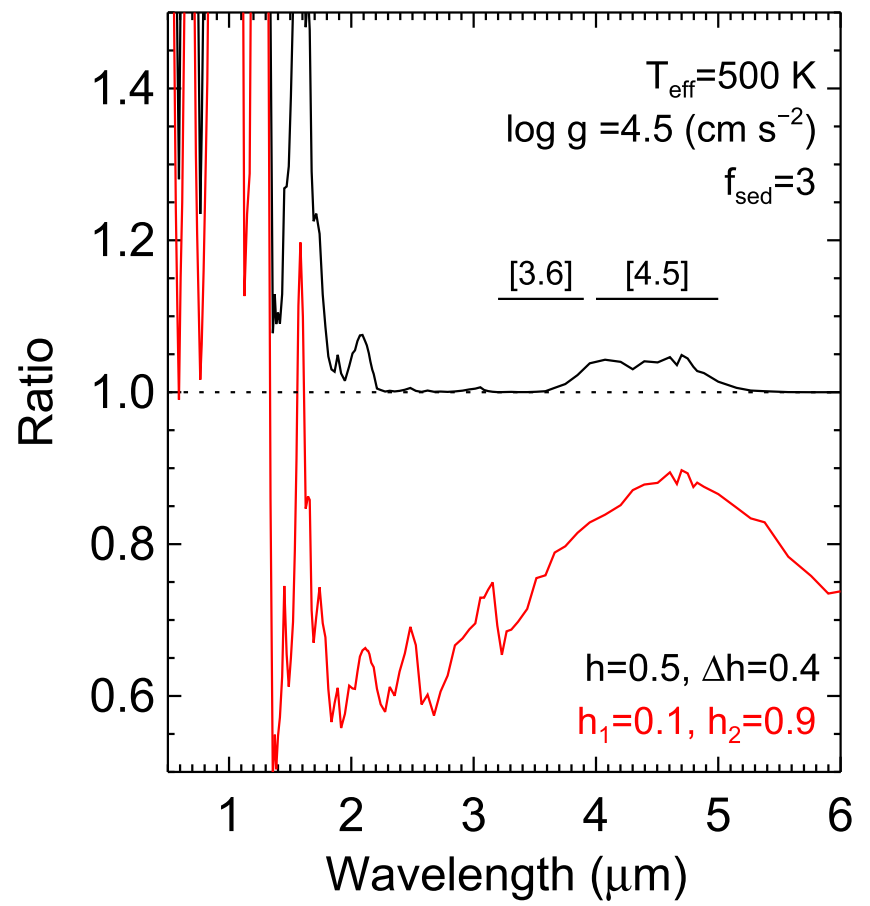

Figure 8. Ratio of model spectra, with $T_{\text {eff }}=500 \mathrm{~K}, \log g=4.5\left(\mathrm{~cm} \mathrm{~s}^{-2}\right)$, $f_{\text {sed }}=3$ computed in two ways. Red: the two spectra were computed independently, with hole fraction values of $h_{1}=0.1$ and $h_{2}=0.9$. Black: the two spectra were computed using a single hole fraction value of $h=0.5$, with $\Delta h=0.4$. The $50 \%$ power points of the IRAC [3.6] and [4.5] bandpasses are also indicated.

(see also Figure 6). Similarly, the models always predicted a larger semi-amplitude at [3.6] instead of [4.5]. Indeed the only model with a semi-amplitude that approaches the observations has $T_{\text {eff }}=500 \mathrm{~K}, \log g=5.0\left(\mathrm{~cm} \mathrm{~s}^{-2}\right), h=0.5$, and $\Delta h=0.5$ and predicts $A_{[3.6]}=0.017$ and $A_{[4.5]}=0.036$.

The stark contrast in the semi-amplitudes predicted by the two methods is more easily understood by plotting the ratios of the model spectra as a function of wavelength instead of focusing on the semi-amplitudes at [3.6] and [4.5]. Figure 8 shows the ratio of model spectra computed with $\left(h_{1}, h_{2}\right)=(0.1,0.9)$ and with $h=0.5, \Delta h=0.4$. The selfconsistent models with $h=0.5, \Delta h=0.4$ result in large ratios at near-infrared wavelengths but small ratios at mid-infrared wavelengths. For these models, the pressure-temperature profile used to calculate the spectra is the same (converged with $h=0.5$ ); the cloud opacity is the only difference between the hemispheres. This means that the differences in flux are due only to the opacity of the clouds limiting the depth from which flux emerges. In contrast, the ratio of two models computed with two different $h$ values $\left(h_{1}, h_{2}\right)=(0.1,0.9)$ show large ratio values across all wavelengths, including decreased flux at longer wavelengths. In these models, the total flux emerging from each hemisphere is held constant (both have an effective temperature of $500 \mathrm{~K}$ ), but the pressure-temperature profiles are different by tens of degrees. The total opacity is lower in the less cloudy model, causing the temperature profile to be cooler than the cloudy model to emit the same total flux. This means that the flux in the near-infrared is higher for the clearer model (due to the lower cloud opacity) and the flux in the mid-infrared is smaller (due to the lower temperature at a given pressure).

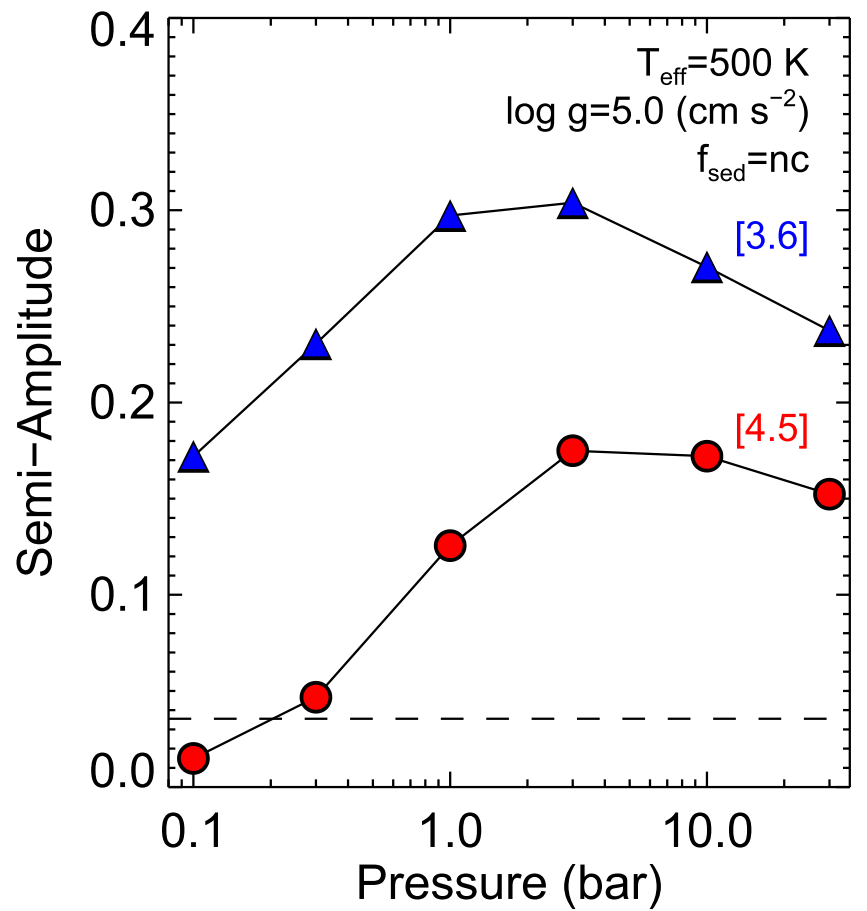

Figure 9. Semi-amplitudes at [3.6] (blue triangles) and [4.5] (red circles) as a function of the depth of the atmosphere at which thermal energy is injected for a cloudless $\left(f_{\text {sed }}=\mathrm{nc}\right)$ model with $T_{\text {eff }}=500 \mathrm{~K}, \log g=5.0\left(\mathrm{~cm} \mathrm{~s}^{-2}\right)$. The average of the near-equal [3.6] and [4.5] semi-amplitudes of the second-epoch light curve of WISE J1405+5534 is shown as a dashed line.

\subsubsection{Hot Spots}

Variability can also be generated by atmospheric dynamics that perturb the temperature structure of an atmosphere. For example, the rising or sinking of air parcels on a timescale that is faster than the time in which the air parcels can equilibrate with their surroundings will produce "hot" or "cold" spots that could modulate the integrated intensity as the object rotates. Vertically propagating temperature perturbations, whether induced by atmospheric waves or simple radiative coupling (e.g., Robinson \& Marley 2014) could also potentially produce localized temperature anomalies. Morley et al. (2014a) simulated the perturbations arising from hot spots by injecting energy $\left(F_{\text {new }}=1.5 F_{\text {baseline }}\right)$ at various pressure levels $(0.1-30$ bars in steps of $\log _{10}(3)$ dex) into static, one-dimensional, and cloudless $\left(f_{\text {sed }}=\infty\right)$ model atmospheres. Model spectra are then generated by assuming that $5 \%$ of the surface is covered by the hot spot. We then determined a synthetic semi-amplitude as a function of the depth of the energy deposition by computing the average flux densities through the Spitzer [3.6] and [4.5] bandpasses for both perturbed and unperturbed cloudless model spectra with $T_{\text {eff }}=500 \mathrm{~K}, \log g=5.0\left(\mathrm{~cm} \mathrm{~s}^{-2}\right)$ and the results are shown in Figure 9.

Perturbations at nearly all depths produce semi-amplitudes that are too large when compared to our observations. Although perturbations at the 0.1 and 0.3 bar level of the atmosphere produced semi-amplitudes that are consistent with our [4.5] observations, they produce variability that is much too large at [3.6]. More troubling, however, is the fact that the hot spot models predict that the [3.6] amplitude is always larger than [4.5] amplitude, i.e., $A_{[3.6]} / A_{[4.5]} \gg 1$. Given that our firstepoch observations have a ratio less than unity and our secondepoch observations have a ratio approximately equal to unity, 
hot spots can probably be ruled out as the underlying cause of the observed variability in WISE J1405+5534.

There are, however, two caveats to this assertion. First, the amount of energy injected at a given layer of the atmosphere was arbitrarily set to 1.5 times the baseline emergent flux for illustrative purposes. Values less than 1.5 produce lower amplitudes that are more inline with our observations, but $A_{[3.6]} / A_{[4.5]}$ always remains greater than unity. Second, we have only performed the calculation with a single, cloudless model. Before hot spots can be definitively ruled out as a possible contributor to the variability in cool brown dwarfs, models at different temperatures and surface gravities that include the formation of clouds must be inspected.

\section{DISCUSSION}

Since the detection of variability in brown dwarfs has so far been limited to the $\mathrm{L}$ and $\mathrm{T}$ dwarfs, it is important to place our observations of WISE J1405+5534 in context with these results. Crossfield (2014) compiled a catalog of late-type M, L, and $\mathrm{T}$ dwarfs with measured rotation periods, $v \sin i$ values, or variability amplitudes. This catalog does not, however, include the recent results of Metchev et al. (2015), who searched $44 \mathrm{~L}$ and T dwarfs (L3-T8) for variability in the mid-infrared with Spitzer. We have therefore combined the Metchev et al. results with the Crossfield catalog and converted peak-to-peak amplitudes to semi-amplitudes when appropriate.

Only four $\mathrm{L}$ and two $\mathrm{T}$ dwarfs have measured rotation periods larger than $8.5 \mathrm{hr}$, making WISE J1405+5534 one of the slower rotating field brown dwarfs known. Indeed, at spectral types later than T3, WISE J1405+5534 has the longest period measured to date. It is, however, currently unclear whether this has physical significance (e.g., brown dwarfs spin down as they cool) or is simply a result of observational bias (e.g., most searches do not have the time baseline to detect such long periods). The [3.6] and [4.5] epoch 2 semi-amplitudes of $3.5 \%$ are also the largest mid-infrared amplitudes observed to date, with 2MASS J22282889-4310262 (T6) coming in second at $2.8 \%$ (Metchev et al. 2015). Of course, this amplitude is still far below that seen in the red-optical and near-infrared, where semi-amplitudes of 5\%-13\% have been reported for some L and T dwarfs (Artigau et al. 2009; Radigan et al. 2012; Gillon et al. 2013).

Metchev et al. also found that the maximum variability amplitude in both the [3.6] and [4.5] bands increases through the $\mathrm{L}$ and $\mathrm{T}$ spectral classes, although the trend beyond $\mathrm{T} 3$ is based on a single $\mathrm{T}$ dwarf (the aforementioned 2MASS J22282889-4310262). They fit a relation to the upper envelope of the [3.6] peak-to-peak amplitudes that predicts a maximum semi-amplitude of $4.25 \%$ for a Y0.5 dwarf. The epoch 2 [3.6] semiamplitude of $3.6 \%$ falls below this prediction and therefore, based on this solitary Y dwarf, the Metchev et al. relation appears to hold across the $\mathrm{T} / \mathrm{Y}$ boundary. The epoch 2 semi-amplitude ratio of $A_{[3.6]} / A_{[4.5]} \approx 1$ for WISE J1405+5534 is also formally consistent with the mean value of 1.0 (with a standard deviation of 0.7 ) for $\mathrm{L}$ and $\mathrm{T}$ dwarfs measured by Metchev et al. (2015). However, without a detection of variability at [3.6] in epoch 1 , it remains unclear just how low the amplitude ratio can be for this object.

Finally, although these first observations of $\mathrm{Y}$ dwarf variability have given us some constraints on the timescales over which variability occurs, we are fundamentally limited in what we can learn about their atmospheres for two reasons.
First, our two mid-infrared wavelengths probe a limited range of atmospheric pressures high in the atmosphere (see Figure 3). In addition, these layers fall well above the expected major $\mathrm{KCl}$ and $\mathrm{Na}_{2} \mathrm{~S}$ cloud decks, which means it is likely that our observations are not directly probing atmospheric layers with clouds. A high water cloud could also be a candidate for the variability in the second epoch because it forms at these high atmospheric layers, but such an explanation also faces difficulties, as our best-fitting models are not cold enough to form an optically thick cloud (see Morley et al. 2014b). Nevertheless, variability arising from water clouds should be considered in further studies. Simultaneous, multi-wavelength observations over a larger wavelength range (i.e., near- and mid-infrared observations) would allow us to study multiple layers of the atmosphere with and without clouds. Second, the models we compare observations to are one-dimensional and static and thus any attempt to compare time-dependent phenomena to them is at some level ad hoc. Two-dimensional or three-dimensional models that include time-dependent phenomena like vertical mixing, cloud formation, and thermal relaxation are therefore sorely needed.

We thank the anonymous referee for comments that improved the paper. This publication makes use of data products from the Wide-field Infrared Survey Explorer, which is a joint project of the University of California, Los Angeles, and the Jet Propulsion Laboratory/California Institute of Technology, funded by the National Aeronautics and Space Administrations and is based [in part] on observations made with the Spitzer Space Telescope, which is operated by the Jet Propulsion Laboratory, California Institute of Technology under a contract with NASA. Support for this work was provided by NASA through an award issued by JPL/Caltech. and the NASA/ESA Hubble Space Telescope, obtained at the Space Telescope Science Institute, which is operated by the Association of Universities for Research in Astronomy, Inc., under NASA contract NAS 5-26555. This research has made use of the NASA/IPAC Infrared Science Archive, which is operated by the Jet Propulsion Laboratory, California Institute of Technology, under contract with the National Aeronautics and Space Administration.

\section{REFERENCES}

Ackerman, A. S., \& Marley, M. S. 2001, ApJ, 556, 872

Artigau, É, Bouchard, S., Doyon, R., \& Lafrenière, D. 2009, ApJ, 701, 1534 Bailer-Jones, C. A. L., \& Mundt, R. 2001, A\&A, 367, 218

Beuzit, J.-L., Feldt, M., Dohlen, K., et al. 2008, Proc. SPIE, 7014, 701418

Buenzli, E., Apai, D., Morley, C. V., et al. 2012, ApJL, 760, L31

Burgasser, A. J., Marley, M. S., Ackerman, A. S., et al. 2002, ApJL, 571, L151 Chamberlain, J. W., \& Hunten, D. M. 1987, in International Geophysics Series,

Vol. 36, Theory of planetary atmospheres. An introduction to their physics and chemistry (Orlando, FL: Academic Press, Inc.)

Cohen, M., Wheaton, W. A., \& Megeath, S. T. 2003, AJ, 126, 1090

Crossfield, I. J. M. 2014, A\&A, 566, A130

Cushing, M. C., Kirkpatrick, J. D., Gelino, C. R., et al. 2011, ApJ, 743, 50

Cushing, M. C., Roellig, T. L., Marley, M. S., et al. 2006, ApJ, 648, 614

Dorren, J. D. 1987, ApJ, 320, 756

Dupuy, T. J., \& Kraus, A. L. 2013, Sci, 341, 1492

Enoch, M. L., Brown, M. E., \& Burgasser, A. J. 2003, AJ, 126, 1006

Fazio, G. G., Hora, J. L., Allen, L. E., et al. 2004, ApJS, 154, 10

Foreman-Mackey, D., Hogg, D. W., Lang, D., \& Goodman, J. 2013, PASP, 125,306

Gelino, C. R., Marley, M. S., Holtzman, J. A., Ackerman, A. S., \& Lodders, K. 2002, ApJ, 577, 433

Gillon, M., Triaud, A. H. M. J., Jehin, E., et al. 2013, A\&A, 555, L5

Heinze, A. N., Metchev, S., Apai, D., et al. 2013, ApJ, 767, 173 
Hillenbrand, L. A., Foster, J. B., Persson, S. E., \& Matthews, K. 2002, PASP, 114,708

Hogg, D. W., Bovy, J., \& Lang, D. 2010, arXiv:1008.4686

Khandrika, H., Burgasser, A. J., Melis, C., et al. 2013, AJ, 145, 71

Kirkpatrick, J. D., Gelino, C. R., Cushing, M. C., et al. 2012, ApJ, 753, 156

Knutson, H. A., Charbonneau, D., Allen, L. E., Burrows, A., \& Megeath, S. T. 2008, ApJ, 673, 526

Marley, M. S., Saumon, D., \& Goldblatt, C. 2010, ApJL, 723, L117

McBride, J., Graham, J. R., Macintosh, B., et al. 2011, PASP, 123, 692

Metchev, S. A., Heinze, A., Apai, D., et al. 2015, ApJ, 799, 154

Mohanty, S., Basri, G., Shu, F., Allard, F., \& Chabrier, G. 2002, ApJ, 571,469
Morales-Calderón, M., Stauffer, J. R., Kirkpatrick, J. D., et al. 2006, ApJ, 653, 1454

Morley, C. V., Fortney, J. J., Marley, M. S., et al. 2012, ApJ, 756, 172

Morley, C. V., Marley, M. S., Fortney, J. J., \& Lupu, R. 2014a, ApJL, 789, L14

Morley, C. V., Marley, M. S., Fortney, J. J., et al. 2014b, ApJ, 787, 78

Radigan, J., Jayawardhana, R., Lafrenière, D., et al. 2012, ApJ, 750, 105

Radigan, J., Lafrenière, D., Jayawardhana, R., \& Artigau, E. 2014, ApJ, 793, 75

Reach, W. T., Megeath, S. T., Cohen, M., et al. 2005, PASP, 117, 978

Robinson, T. D., \& Marley, M. S. 2014, ApJ, 785, 158

Schneider, A. C., Cushing, M. C., Kirkpatrick, J. D., et al. 2015, ApJ, 804, 92

Showman, A. P., \& Kaspi, Y. 2013, ApJ, 776, 85 